\title{
Music Intervention in Undergraduates: the Relationship between Heart Rate Variability and State Anxiety
}

\author{
Annalise J. Tolley* \& Robert S. Vick \\ Department of Biology, Elon University, Elon, NC \\ bttps:/ / doi.org/10.33697/ ajur.2020.003 \\ Student:atolley2@elon.edu* \\ Mentor:vickrs@elon.edu
}

\begin{abstract}
A low heart rate variability (HRV) is indicative of autonomic inflexibility, which has important implications for physical and psychological health. This study investigates autonomic functioning and its relationship to state anxiety in the context of music intervention. A within-subjects, quasi-experimental design was used with undergraduates, a population frequently impacted by state anxiety. Participants pre-selected music that they identified as the most "relaxing" before being administered the State-Trait Anxiety Inventory (STAI). Baseline physiological indices were then collected using an EKG, followed by administration of the selected music and an additional collection of physiological indices. After the full duration of music intervention was administered, participants took the STAI once more. Results indicated that even short durations of music listening may serve to reduce anxiety in the undergraduate student, as was evidenced by the significant increase in physiological indices and decrease in self-reported anxiety levels. However, while changes in HRV and anxiety may trend together, results indicated no statistical association between these parameters.
\end{abstract}

\section{KEYWORDS}

Heart Rate Variability; Anxiety; Music Intervention; Autonomic Tone; Vagal Control; Autonomic Flexibility; College Students; State Anxiety

\section{INTRODUCTION}

State Anxiety in the Undergraduate

Undergraduates face a variety of stressors in their lives. Their competitive academics, leadership roles, volunteer positions, and extracurriculars are just a few of the many commitments that contribute to a hectic schedule. While all of these expectations may seem benign, statistics reveal a more disconcerting picture. Anxiety in undergraduates is on the rise, with almost $60 \%$ of students in 2016 reporting overwhelming anxiety in the past 12 months and 85\% reporting being overwhelmed by all that had to be done. ${ }^{1}$ Close to $50 \%$ of students also reported that their academics felt "traumatic or very difficult to handle." 1 While only $17 \%$ of the students surveyed were diagnosed with anxiety by a medical professional, ${ }^{1}$ it is clear that these feelings of sub-clinical anxiety are distressing to the individual and deserve attention. As such, diagnosis or not, the mental health of undergraduates is increasingly in jeopardy.

Anxiety can be classified as either state anxiety or trait anxiety. ${ }^{2}$ Trait anxiety refers to personality-driven anxiety. This is the general tendency for someone to perceive the environment as threatening, and it is relatively constant. State anxiety, on the other hand, is subjective and fleeting. It is a psychological and physiological response to situational feelings of tension. ${ }^{3}$ Given the large proportion of undergraduates who report feelings of anxiety and tension without qualifying for diagnosis, these individuals are presumably feeling the effects of situational, state anxiety. From this point forward, unless otherwise specified, the use of the word anxiety refers to anxiety at large, rather than a clinical diagnosis.

\section{Measuring Anxiety Through Autonomic Flexibility}

Various self-reported measures exist to determine anxiety, but research has also provided evidence that anxiety can be measured physiologically using heart rate variability (HRV). ${ }^{4-7} \mathrm{HRV}$ is a physiological indicator of autonomic/ vagal tone, which is the interplay between the sympathetic and parasympathetic branches of the autonomic nervous system (ANS). Vagal tone has long been accepted as an indicator of cardiac and physical health, ${ }^{8,9}$ with low vagal tone correlating to diabetes, ischemic heart failure, and early mortality. 8,9 Low vagal tone is an indicator of poor health because it is a measurement of poor autonomic flexibility. High autonomic flexibility is an adaptive trait allowing for organismic stability in the face of a complex, dynamic environment. ${ }^{5,10}$ 
Measuring autonomic flexibility through vagal tone provides important ties to other forms of flexibility, such as psychological flexibility. ${ }^{11,12}$ Vagal control is unique because it integrates both the ANS and the CNS via the central autonomic network (CAN), which is mediated by the vagal nerve. ${ }^{5,13}$ The CAN provides a pathway for autonomic tone to reflect and impact behavioral processes and vice versa. It is the mechanistic explanation for the impact of vagal tone on mental health and is the basis of the autonomic flexibility-neurovisceral integration model of anxiety. This model states that low vagal tone is a predictor of all forms of anxiety due to a rigid inability to dis-inhibit negative feedback mechanisms both physiologically and psychologically. ${ }^{5}$ If this model is accepted as true, HRV (as a measurement of vagal tone) measures not only autonomic flexibility but psychological flexibility as well. Relevant to this study, lack of psychological flexibility can present as anxiety, which is the inability to dis-inhibit an inappropriate response following a benign stimulus. ${ }^{14}$

\section{The Present Study}

To further explore this psychophysiological model of anxiety, investigators of this study included music intervention. Research indicates that music can alter both psychological and autonomic states, ${ }^{14-18}$ although the exact mechanism of music's effect on the ANS is not entirely known. ${ }^{14,19,20}$ Music intervention served a primary purpose in this study: to function as a situational stimulus that could decrease state anxiety as measured by self-report and physiological indices. Any resultant changes in parameters would then be compared. If psychological and physiological anxiety are integrated to the degree that the autonomic flexibility-

neurovisceral integration model theorizes, then comparison should yield a significant relationship. This finding would support that HRV is, in fact, a psychophysiological measurement rather than just a physiological one.

Other studies investigating music intervention and its effects on state anxiety have shown promising results that provide reason to believe it may be effective for undergraduates. ${ }^{15,16,18}$ While not the primary goal of the study, if the hypothesis of this study were confirmed, results may imply that music intervention is a brief and efficacious method for reducing state anxiety in the undergraduate.

In summation, the present study seeks to determine the extent of the possible relationship between anxiety and HRV by using music intervention. Based on the literature, the study is conducted with the assumption that STAI score is a measurement of psychological anxiety, and HRV is a measurement of physiological anxiety. It is hypothesized that (1) music intervention will lead to decreased psychological and physiological anxiety, as measured through STAI score and HRV indices, respectively; and (2) the changes in psychological and physiological anxiety will be related to one another.

\section{METHODOLOGY}

\section{Participants}

Healthy undergraduate students at Elon University $(\mathrm{n}=32)$ were recruited to participate (mean age 19.2 yrs., range 17-22 yrs.). Participants were not screened for anxiety before participating; there were no exclusion criteria. Previous sample sizes in the field and prior sample size calculations provide basis to believe that 32 participants may allow for adequate assessment $(\beta=0.8)$ of a medium to large effect size. ${ }^{18}$ However, across studies, sample size calculations are inconsistent. This study was approved by the Elon University's Institutional Review Board (\#17-083). The purpose of the study, methods, potential risks, and benefits were outlined to the participants by an informed consent form. The participants' rights and privacy were protected throughout the experiment, and participants had the right to withdraw from the study at any given time.

\section{Study Design}

All participants were part of both the pre- and post-experimental conditions to control for individual differences. To control for HRV variation as a result of circadian rhythm, which differs throughout the day, ${ }^{21}$ data collection occurred within the span of $5 \mathrm{pm}$ to $6 \mathrm{pm}$. After written consent was obtained, the State-Trait Anxiety Inventory (STAI) was administered through pencil and paper to the subjects. After the survey was completed, the participants were played a sample from four preselected songs and asked to choose the most relaxing song option, which would be played in the latter part of the experiment. The subjects were then attached to an EKG, and seven minutes of baseline HRV data was collected. The pre-selected music was then played for an additional seven minutes as HRV data continued to be collected. After the fourteen minutes of EKG data collection, participants were asked to complete the STAI Y-1 once again. The total experiment duration was 30 minutes.

\section{Psychological Measure}

To measure anxiety, the State-Trait Anxiety Inventory (Y-1 and Y-2) was used. A leading limitation in anxiety research thus far has been the surveys used for evaluation. Very few tests have the ability to differentiate between depression and anxiety, ${ }^{22}$ and due to this methodological flaw, much of the research that explores anxiety and HRV may very well be an extension of preexisting HRV and depression research. ${ }^{23}$ The State-Trait Anxiety Inventory (STAI) have been shown to better distinguish anxiety with high reliability and validity across different languages, cultures, and populations.22, 24-26 The STAI includes two 20-questions surveys, in which the participant is asked to rate their response on a Likert 1-4 scale. The Y-1 measures state anxiety (anxiety 
attributed to external stimulus), and the Y-2 measures trait anxiety (anxiety attributed to personality). The average alpha reliability coefficients for college students were 0.92 and 0.91 on the Y-1 and Y-2, respectively. ${ }^{2}$ A higher score indicates more anxiety, and it is thought that scores below 40 are not considered clinically significant symptoms. ${ }^{22}$

\section{Physiological Measure}

HRV was monitored and measured with lead I of a three-lead EKG. Electrodes were connected to a computer-based data acquisition system (ADInstruments, Boulder, CO) and analyzed using Lab Chart Pro and its HRV module (ADInstruments).

\section{Acoustic Stimuli}

This study offered participants four options for music intervention, and participants were asked to choose which song they found most "relaxing." In preexisting studies, participants have been allowed to self-select music used for their intervention, ${ }^{20}$ which leaves room for a myriad of confounding variables. To reduce these confounding effects without completely removing the autonomy of the participant, subjects were given four musical genre options (jazz, orchestral, elemental healing sounds, and instrumental pop) controlled for tempo $(60 \mathrm{bpm}),{ }^{20}$ lyrics (no lyrics), ${ }^{27}$ and relative volume. ${ }^{17}$ Because the aim of the study was to measure whether or not music could lower psychological and physiological measures of state anxiety in subjects, it was important to avoid accidental arousal of the individual via PNS withdrawal or subjective dislike due to the song. ${ }^{17,20}$ Of the four available options, subjects were able to choose the most subjectively relaxing choice. Research has shown that maintaining options in music intervention studies is important for both the subjects and the experimental design. ${ }^{16}$

\section{Statistical Analysis}

IBM SPSS Statistics 24 was used for data analysis. Descriptive statistics were run, and data was normally distributed. A paired ttest was used to evaluate if there were differences between the STAI scores and HRV indices pre- and post- music administration. Principal components analysis was used to derive a composite HRV index, which was then compared using repeated measures ANOVA to STAI score. Significance was set at $\mathrm{p}<0.05$ for all analyses.

\section{RESULTS}

Psychological Measure

For STAI score, the Y-1 score (state anxiety) was used in analysis and the Y-2 (trait anxiety) was not. Because the Y-2 is representative of general, personality-related anxiety, it would not be expected to change based on external stimuli. It therefore was only administered once. A one-way, unpaired t-test between individuals with clinically significant STAI scores (above 40 on Y-2) and non-clinically significant anxiety indicated no significant difference between any of the HRV indices. As a result, Y-2 was not used at this time for data analysis.

The STAI Y-1 score decreased for 31 out of 32 of the participants. To test if the overall reduction in Y-1 score was significant, a one-way, paired t-test was run. Figure 1 illustrates the results: a significant reduction in Y-1 score $(p<0.0001)$ from an average STAI score of $38 \pm 8.87$ before music administration to $29 \pm 5.98$ after music administration.

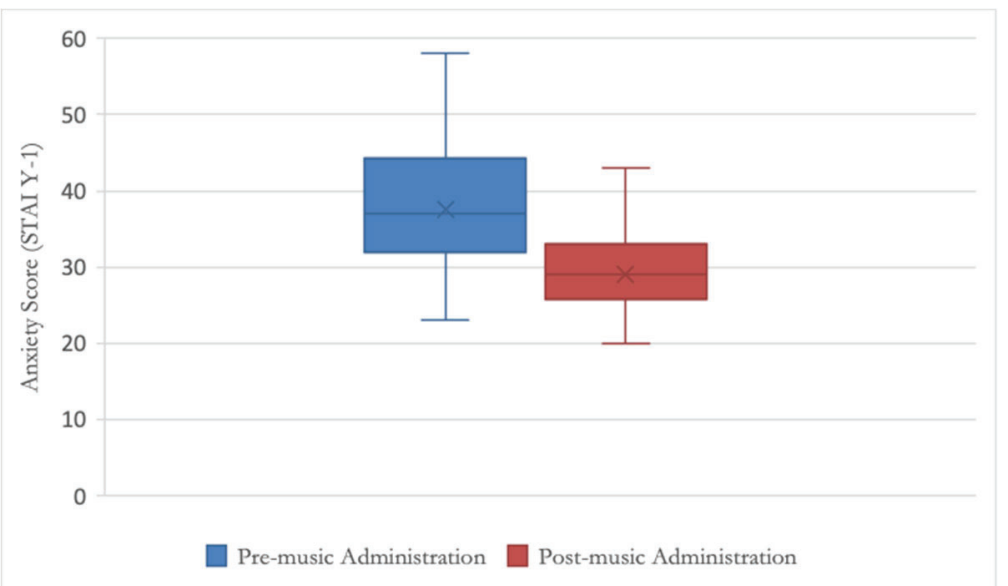

Figure 1. Average STAI score before and after music administration. Error bars are SEM ( $\mathrm{n}=32)$. 


\section{Physiological Measure}

In addition to the STAI score, average RR, SDRR, pRR50, and HR were collected both pre- and post-music administration. RR is the time interval measurement from one $\mathrm{R}$ peak to the next in milliseconds ( $\mathrm{ms}$ ). The SDRR is standard deviation of all RR measurements (ms). The pRR50 can be thought of as the percentage of RR interval differences greater than $50 \mathrm{~ms}$ from the previous RR. HR is average heart rate (bpm). The data analysis in this experiment used time domain analysis. There was high variation in HRV range based on the individual. To both account for this and exclude ectopic beats, the data was plotted and the range was adjusted to include the majority of heart beats. HRV intervals for each participant were kept within 400 ms and ranged from $400-800 \mathrm{~ms}$ to $800-1200 \mathrm{~ms}$.

Paired t-tests were used to analyze physiological indices before and after music intervention. Results indicated that average RR increased from $743.08 \pm 122.71 \mathrm{~ms}(\mathrm{p}<0.001)$ to $759.5 \pm 123.78 \mathrm{~ms}$, SDRR increased from $51.84 \pm 25.29 \mathrm{~ms}$ to $57.27 \pm 24.62$ $\mathrm{ms}(\mathrm{p}<0.01)$, pRR50 increased from $17.29 \pm 17.9 \%$ to $20.61 \pm 20.3 \%$ ( $<<0.01)$, and average HR decreased from $83.19 \pm 12.78$ $\mathrm{bpm}$ to $81.46 \pm 12.70 \mathrm{bpm}(\mathrm{p}<0.001)$. Participants showed a statistically significant difference in HRV indices after music administration. This difference reflected physiological relaxation after music administration, as determined by an increased RR, SDRR, and pRR50 and a decrease in HR, is reflected in Table 1.

\begin{tabular}{|c|c|c|c|}
\hline & Pre-music & Post-music & $p$-value \\
\hline Average RR (ms) & 743.1 & 759.5 & $<0.005$ \\
\hline SDRR (ms) & 51.84 & 57.27 & $<0.005$ \\
\hline pRR50 (\%) & 17.29 & 20.61 & $<0.005$ \\
\hline Average HR (bpm) & 83.19 & 81.46 & $<0.005$ \\
\hline
\end{tabular}

Table 1. Average HRV indices pre- and post-music administration. The p-values were determined with one-tailed, paired t-tests.

Psychological and Physiological Measure

Although results indicate that both STAI scores and HRV indices are significantly different after music administration, to determine the extent to which these variables may be related, a Pearson's correlation was run between STAI and each HRV index both pre- and post-music administration. It was determined that there was no significant correlation between anxiety and average $\mathrm{RR}([\mathrm{pre}] \mathrm{r}=-0.11, \mathrm{p}=0.95 ;$ [post $] \mathrm{r}=0.03 ; \mathrm{p}=0.87)$, anxiety and SDRR $(\mathrm{r}=0.01, \mathrm{p}=0.98 ; \mathrm{r}=-0.18 ; \mathrm{p}=0.34)$, anxiety and $\mathrm{pRR} 50(\mathrm{r}=0.14, \mathrm{p}=0.45 ; \mathrm{r}=-0.04 ; \mathrm{p}=0.82)$, or anxiety and HR $(\mathrm{r}=0.02, \mathrm{p}=0.90 ; \mathrm{r}=-0.46 ; \mathrm{p}=0.80)$, which is summarized in Table 2.

\begin{tabular}{|c|c|c|c|}
\hline \multicolumn{2}{|c|}{ Pearson's Correlation } & P-value \\
\hline \multirow{2}{*}{ Variables } & Treatment & -0.11 & 0.95 \\
\hline \multirow{2}{*}{ SDRR v. STAI } & Pre & 0.03 & 0.87 \\
\cline { 2 - 4 } & Post & 0.01 & 0.98 \\
\cline { 2 - 4 } pRR50 v. STAI & Pre & -0.18 & 0.34 \\
\cline { 2 - 4 } & Post & 0.14 & 0.45 \\
\hline \multirow{2}{*}{ HR v. STAI } & Pre & -0.46 & 0.82 \\
\cline { 2 - 4 } & Post & 0.02 & 0.90 \\
\hline
\end{tabular}

Table 2. Pearson's correlation table between HRV indices and anxiety measure.

Because Pearson's correlation is most often used to investigate the relationship between either an independent variable and a dependent variable or two factorials, principal component analysis (PCA) was used to explore the relationship between the five dependents. The first run of PCA yielded a correlation matrix that showed no significant correlation between STAI score and RR, SDRR, pRR50, and HR with $r$-values respectively as follows: $r=-0.28, r=-0.110, r=0.008, r=0.030$. After STAI was removed, PCA was again run. Table 3 shows the produced correlation matrix and the scree plot, which were used to identify relevant component PC1.

The PC1 was used as the composite variable of HRV indices and a univariate ANOVA was run to confirm a statistically significant physiological difference after music intervention. The average PC1 pre-music was $-0.092 \pm 1.01$ and the average PC1 post-music was $0.092 \pm 1.00$. A statistically significant difference was confirmed using a paired sample t-test $(\mathrm{p}<0.005)$. A negative PC1 value, as is the value pre-music, corresponds to an anxious physiological state, as measured by a high HR, low average RR, low SDRR, and low pRR50. Conversely, a positive PC1 value, as in the post-music score, would correspond to a 
relaxed physiological state, as measured by a lower HR, higher average RR, higher SDRR, and higher pRR50. Results indicate that after music administration, participants were more physiologically relaxed than before music administration.

A Pearson's correlation was once more run but this time between STAI score and PC1 pre- and post-music administration. No statistically significant correlation was found between STAI score and PC1 $(\mathrm{r}=-0.043, p=0.737)$.

\begin{tabular}{|c|c|c|c|c|c|}
\hline \multicolumn{2}{|c|}{} & RR & SDRR & pRR50 & HR \\
\hline Correlation & RR & 1.000 & $0.592^{*}$ & $0.741^{*}$ & $-0.981^{*}$ \\
\cline { 2 - 6 } & SDRR & 0.592 & 1.000 & $0.784^{*}$ & $-0.555^{*}$ \\
\cline { 2 - 6 } & pRR50 & 0.741 & 0.784 & 1.000 & $-0.711^{*}$ \\
\cline { 2 - 6 } & HR & -0.981 & -0.555 & -0.711 & 1.000 \\
\hline
\end{tabular}

Table 3. PCA correlation matrix of HRV indices with excluded STAI score. Relevant correlations are starred.

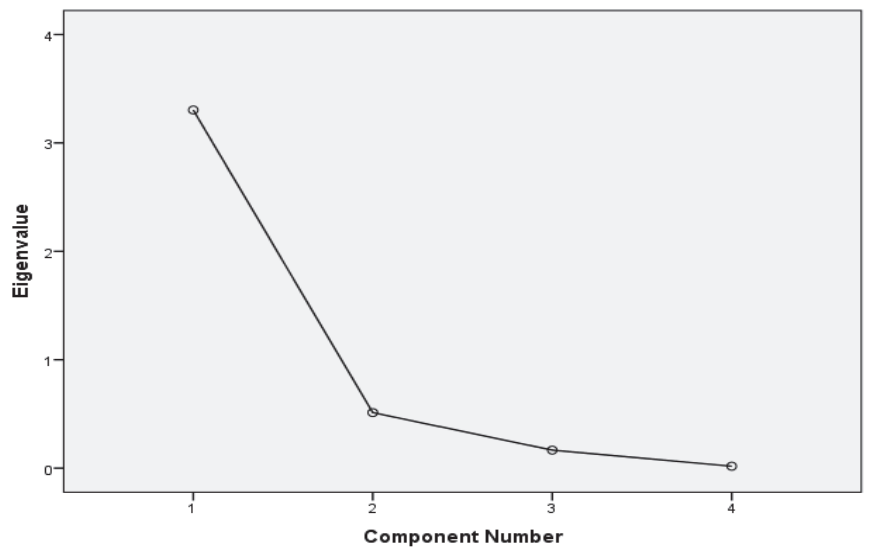

Figure 2. Scree plot of HRV components. Following the broken stick model, the relevant component was PC1.

\section{DISCUSSION}

It was hypothesized that (1) music intervention would lead to decreased psychological and physiological anxiety, as measured through STAI score and HRV indices, respectively; and (2) the changes in psychological and physiological anxiety would be related to one another, as determined by statistical significance.

Results supported hypothesis one and indicated that relaxing music intervention resulted in a statistically significant decrease in self-reported state anxiety, as measured by the STAI Y-1. These findings support previous research on the positive effects of music intervention on psychological and physiological measures. ${ }^{19,}{ }^{28}$ However, many of these studies administer music for $20-30$ minutes. This study shortened the duration of administered music to seven minutes, which is more manageable for situational intervention given the busy schedule of the undergraduate. Results indicated a significant benefit to undergraduates from just seven minutes of music, lowering state anxiety by $23 \%$ on average. Thus, the use of music intervention to lower state anxiety, even for a few minutes a day, should be encouraged to the undergraduate population who greatly suffers from daily anxiety. ${ }^{1}$ Because this study investigated the lowered state anxiety at baseline, it would be interesting to further investigate the persistence of this effect. Could a lowered state anxiety mitigate the anxiety-inducing effects of a situational stressor? If so, this could have practical implications for the undergraduate, who often knows when situational stressors will occur, such as a test or presentation.

In addition to a significant decrease in self-reported anxiety, results further support hypothesis one: music intervention would result in increased HRV indices, a reflection of reduced physiological anxiety. Although there is little research on the effect of music specifically on HRV, there is a breadth of literature that examines the effect of music on other physiological indices related to autonomic functioning. ${ }^{17,29,30}$ Research has been inconclusive, but this study hypothesized that with the administration of controlled, "relaxing" music, there would be a significant effect on autonomic modulation as measured by HRV, which was supported by the results. PC1, the component representative of all the HRV indices, significantly shifted in the positive direction after music intervention. A positive shift reflected a decrease in physiological anxiety through an increase in HRV, as measured by higher RR, SDRR, pRR50, and lower HR. 
Hypothesis two postured that the significant changes in HRV and STAI score would be statistically correlated to one another based on the autonomic flexibility-neurovisceral integration model of anxiety. This was unsupported. While results indicated that both dependents changed significantly in the predicted direction after music intervention, further analysis revealed that the changes in psychological and physiological anxiety were not statistically correlated to each other, as measured by Pearson's correlation. Since both Pearson's correlation and correlation matrices assume a linear relationship between variables, it is possible that the changes in HRV and anxiety were not linearly related. However, graphs between STAI and PC1 as well as STAI and HRV individual indices indicated no clear trend to fit the data. Thus, while it is likely that HRV and anxiety trend together, this study found no empirical base to support a significant relationship. As such, HRV may only be a reliable measurement of physiological anxiety, rather than a psychophysiological measurement of anxiety. If this were the case, future research should take caution using HRV as a measurement of anxiety independent of self-report.

Although state anxiety and trait anxiety are somewhat related, correlations between the state and trait anxiety for college students has varied from 0.11 to 0.53 for females and 0.37 to 0.67 for males. ${ }^{2}$ Because of this variability, when conclusions are drawn, they should not be extrapolated to anxiety in general but rather to the specific anxiety being measured. This study's design has implications only for state anxiety, which is anxiety produced from situation, not disposition. It is therefore possible that HRV is more indicative of trait anxiety, but not state anxiety.

Several limitations exist, and results should be interpreted modestly. These limitations include the generalizability of the data, given the minimal diversity of the subjects collected and small sample size. Because subjects were recruited from Elon University, Caucasian females were overrepresented, while males and minority groups were underrepresented. Previous sample sizes in the field and prior sample size calculations provide basis to believe that 32 participants may allow for adequate assessment $(\beta=0.8)$ of a medium to large effect size. ${ }^{18}$ However, across studies, sample size calculations are inconsistent. This poses the risk that many studies in the field, including this one, do not qualify for adequate statistical power.

To reduce the effects of individual differences and account for the high degree of individual variability associated with HRV, a within-subjects, pretest/posttest design was employed. It is worth noting that it is possible that the musical pieces chosen may have had unknown effects on autonomic tone for different individuals. This study tried to maintain a sense of choice for the participants while holding constant variables known to affect autonomic tone and perceptual experience (i.e. lyrics, tempo, volume, genre), ${ }^{17,20,27}$ but it is possible that the subjective perception of music could alter an individual's ANS unknowingly. Likewise, it is possible that not all participants found the music subjectively relaxing.

The aim of this study was to develop a deeper understanding of the possible relationship between psychological state anxiety and physiological anxiety, as measured by STAI and HRV respectively. The hypothesis that music intervention would both lower selfreported anxiety scores and increase HRV was supported, which provides evidence that brief music intervention is a successful tool to lower state anxiety in undergraduate students and to promote autonomic relaxation. The prediction that the change in psychological anxiety (STAI score) and that the change in physiological anxiety (HRV indices) would be statistically correlated was unsupported. Therefore, despite the significant effect that music had on both autonomic functioning and self-reported anxiety, these effects seem only to trend together, as they are not statistically related. While these findings strengthen the potential of music to reduce state anxiety and promote autonomic relaxation in the undergraduate, they also suggest that interpreting HRV as a measurement of something other than physiological anxiety should be approached with caution. Physiological anxiety may trend, even interface, with psychological state anxiety, but not to a significant degree. This may qualify the broader implications of HRV as a measurement of state anxiety.

\section{ACKNOWLEDGEMENTS}

The author thanks Dr. Vick for his critical support and contribution to this study as a whole and to this manuscript. Additionally, the author thanks the staff of the Elon Biology Department for their mentorship and guidance. This research would not have been possible without funding from the Undergraduate Research Program and the Elon College Fellow's program.

\section{DISCLOSURE STATEMENT}

There are no conflicts of interest to disclose.

\section{REFERENCES}

1. American College Health Association: National College Health Assessment. (2016) Fall 2016 Reference Group Executive Summary (Reference Group Executive Summary) (p. 14).

2. Spielberger, C. D., Gorsuch, R. L., Lushene, R., Vagg, P. R., and Jacobs, G. A. (1983) Manual for the State-Trait Anxiety Inventory, Consulting Psychologists Press, Palto Alto, CA. 
3. Horikawa, M., and Akihiro, Y. (2012) The relationships among trait anxiety, state anxiety and the goal performance of penalty shoot-out by university soccer players, PloS One, 7(4). https:// doi.org/10.1371/journal.pone.0035727

4. Chalmers, J. A., Quintana, D. S., Abbott, M. J.-A., and Kemp, A. H. (2014) Anxiety disorders are associated with reduced heart rate variability: A meta-analysis, Front Psychiatry, 5(80). https:// doi.org/10.3389/fpsyt.2014.00080

5. Friedman, B. H. (2007) An autonomic flexibility-neurovisceral integration model of anxiety and cardiac vagal tone, Biol Psychol 74(2), 185-199. https:// doi.org/10.1016/j.biopsycho.2005.08.009

6. Sharma, R.K., Yatan, P. S. B., Sagar, R., Deepak, K. K., and Mehta, M. (2011) Heart rate variability study of childhood anxiety disorders, J Cardiovasc. Dis. Res. 2(2), 115-122. https:/ / doi.org/ http:/ / dx.doi.org/ 10.4103/0975-3583.83040

7. Watkins, L. L., Grossman, P., Krishnan, R., and Sherwood, A. (1998) Anxiety and vagal control of heart rate, Psychosom Med 60(4), 498-502. https:// doi.org/10.1097/00006842-199807000-00018

8. Stein P. K., Kleiger R. E. (1999) Insights from the study of heart rate variability. Annu Rev Med 50, $249-261$. https:// doi.org/ 10.1146/ annurev.med.50.1.249

9. Shigetoh, Y., Adachi, H., Yamagishi, S., Enomoto, M., Fukami, A., Otsuka, M., Kumagae, S., Furuki, K., Nanjo, Y., Imaizumi, T. (2009) Higher heart rate may predispose to obesity and diabetes mellitus: 20-year prospective study in a general population, Am J Hypertens 22(2), 151-155. https:/ / doi.org/10.1038/ajh.2008.331

10. Lloyd, D., Miguel. A. A., and Cortassa, S. (2001) Why homeodynamics, not homeostasis?, Sci World J 1, $133-145$. https:// doi.org/10.1100/tsw.2001.20

11. Mori, K., and Iwanaga, M. (2014) Resting physiological arousal is associated with the experience of music-induced chills, Int J Psychophysiol 93(2), 220-226. https:/ / doi.org/10.1016/j.ijpsycho.2014.05.001

12. Friedman, B. H., and Thayer, J. F. (1998) Anxiety and autonomic flexibility: A cardiovascular approach, Biol Psychol 49(3), 303323. https:/ / doi.org/ 10.1016/S0301-0511(97)00027-6

13. Porges, S. W. (2009) The polyvagal theory: New insights into adaptive reactions of the autonomic nervous system, Cleve Clin J Med 76(Suppl 2), S86-90. https:// doi.org/10.3949/ccjm.76.s2.17

14. Ellis, R. J., and Thayer, J. F. (2010) Music and autonomic nervous system (dys)function, Music Percept 27(4), 317-326. bttps://doi.org/10.1525/mp.2010.27.4.317

15. Bradt, J., and Dileo, C. (2009) Music for stress and anxiety reduction in coronary heart disease patients, Cochrane Database Syst Rev (2). https://doi.org/10.1002/14651858.CD006577.pub2

16. Cooke, M., Chaboyer, W., and Hiratos, M. A. (2005) Music and its effect on anxiety in short waiting periods: a critical appraisal, J Clin Nurs 14(2), 145-155. https:/ / doi.org/10.1111/j.1365-2702.2004.01033.x

17. do Amaral, J. A. T., Guida, H. L., Vanderlei, F. M., Garner, D. M., Osório, E., de Abreu, L. C., and Valenti, V. E. (2015) The effects of musical auditory stimulation of different intensities on geometric indices of heart rate variability, Altern Ther Health Med 21(5), 16-23.

18. Wang, S.-M., Kulkarni, L., Dolev, J., and Kain, Z. N. (2002) Music and preoperative anxiety: A randomized, controlled study, Anesth Analg 94(6), 1489-1494. https:/ / doi.org/10.1213/00000539-200206000-00021

19. Wu, P.-Y., Huang, M.-L., Lee, W.-P., Wang, C., and Shih, W.-M. (2017) Effects of music listening on anxiety and physiological responses in patients undergoing awake craniotomy, Complement Ther Med 32, 56-60. bttps://doi.org/10.1016/j.ctim.2017.03.007

20. Nilsson, U. (2008) The anxiety- and pain-reducing effects of music interventions: A systematic review, AORN J 87(4), 780807. https:// doi.org/ http:/ / dx.doi.org/10.1016/j.aorn.2007.09.013

21. Guo, Y,i-F., and Stein, P. K. (2003) Circadian rhythm in the cardiovascular system: Chronocardiology, Am Heart J 145(5), 779786. https:// doi.org/10.1016/S0002-8703(02)94797-6

22. Julian, L. J. (2011) Measures of Anxiety, Arthritis Care Res, 63(0 11). https:/ / doi.org/ 10.1002/acr.20561

23. Buckby, J. A., Yung, A. R., Cosgrave, E. M., and Killackey, E. J. (2007) Clinical utility of the Mood and Anxiety Symptom Questionnaire (MASQ) in a sample of young help-seekers, BMC Psychiatry 7(50). https:// doi.org/10.1186/1471-244X-7-50

24. Hishinuma, E. S., Miyamoto, R. H., Nishimura, S. T., Goebert, D. A., Yuen, N. Y. C., Makini, G. K., Andrade N. N., Johnson, R. C., and Carlton, B. S. (2001) Prediction of anxiety disorders using the State-Trait Anxiety Inventory for multiethnic adolescents, J Anxiety Disord 15(6), 511-533. https:/ / doi.org/ 10.1016/S0887-6185(01)00079-2

25. Vitasari, P., Wahab, M. N. A., Herawan, T., Othman, A., and Sinnadurai, S. K. (2011) Re-test of State Trait Anxiety Inventory (STAI) among engineering students in Malaysia: Reliability and validity tests, Procedia Soc Behav Sci 15, 3843-3848. https:// doi.org/10.1016/j.sbspro.2011.04.383

26. Zeleníková, R., Homzová, P., Homza, M., and Bužgová, R. (2017) Validity and reliability of the Czech version of the Amsterdam Preoperative Anxiety and Information Scale (APAIS), J Perianesth Nurs 32(5), 429-437. https:// doi.org/10.1016/j.jopan.2016.03.007

27. Ribeiro, M. K. A., Alcântara-Silva, T. R. M., Oliveira, J. C. M., Paula, T. C., Dutra, J. B. R., Pedrino, G. R., Simões, K., Sousa, R. B., and Rebelo, A. C. S. (2018) Music therapy intervention in cardiac autonomic modulation, anxiety, and depression in mothers of preterms: Randomized controlled trial, BMC Psychol 6(57). https://doi.org/10.1186/s40359-018-0271-y 
28. Sendelbach, S. E., Halm, M. A., Doran, K. A., Miller, E. H., and Gaillard, P. (2006) Effects of music therapy on physiological and psychological outcomes for patients undergoing cardiac surgery, J Cardiovasc Nurs 21(3), 194-200. bttps:/ / doi.org/10.1097/00005082-200605000-00007

29. Okada, K., Kurita, A., Takase, B., Otsuka, T., Kodani, E., Kusama, Y., Atarashi, H., and Mizuno, K. (2009) Effects of music therapy on autonomic nervous system activity, incidence of heart failure events, and plasma cytokine and catecholamine levels in elderly patients with cerebrovascular disease and dementia, Int Heart J 50(1), 95-110. bttps:/ / doi.org/ 10.1536/ibj.50.95

30. Phipps, M. A., Carroll, D. L., and Tsiantoulas, A. (2010) Music as a therapeutic intervention on an inpatient neuroscience unit, Complement Ther Clin Pract 16(3), 138-142. https:/ / doi.org/10.1016/j.ctcp.2009.12.001

\section{ABOUT STUDENT AUTHOR}

Annalise J. Tolley graduated from Elon University in May of 2018 with a Bachelor of Science in biology, a concentration in Foundations for Medical Sciences, and a minor in psychology. She has recently completed a one-year fellowship in India working for a public health NGO that works with communities to provide health services, promote empowerment, and enable sustainable development among the poor and marginalized. After refining her research interests over the next year, she intends to pursue a PhD in Social Psychology.

\section{PRESS SUMMARY}

Research suggests that the number of heart beats per minute might be less significant as a determinant for overall health than the interval spacing of those beats. Heart rate variability (HRV) measures the variation in time interval from one beat to the next. A lower HRV indicates less flexibility in a dynamic environment, which is believed to indicate poor physical and mental health. Current theories of anxiety suggest that HRV may be a psychophysiological measure of anxiety, although the degree to which the psychological and the physiological manifestations of anxiety interact need further study. This study explored this possible relationship by measuring changes in psychological anxiety (as measured by State-Trait Anxiety Inventory) and physiological anxiety (as measured by HRV) in response to music intervention. These changes were then compared. Undergraduates were chosen as the population to investigate this trend, as they are often subject to changes in state anxiety and could benefit from brief and accessible intervention. Results indicated that even short durations of music listening reduce anxiety in undergraduates, as was evidenced by the significant decrease in self-reported state anxiety and increase in HRV indices. However, results indicated no statistical correlation between these two parameters. These results may qualify the application of HRV as a psychophysiological indicator of anxiety. 